\title{
Motion Equation and Solution of Mushroom Cloud
}

\section{Tianquan Yun}

School of Civil Engineering and Transportation, South China University of Technology, Guangzhou, China

Email: cttqyun@scut.edu.cn

How to cite this paper: Yun, T.Q. (2021)

Motion Equation and Solution of $\mathrm{Mu}$ shroom Cloud. Atmospheric and Climate Sciences, 11, 86-97.

https://doi.org/10.4236/acs.2021.111006

Received: November 17, 2020

Accepted: January 1, 2021

Published: January 4, 2021

Copyright $\odot 2021$ by author(s) and Scientific Research Publishing Inc. This work is licensed under the Creative Commons Attribution International License (CC BY 4.0).

http://creativecommons.org/licenses/by/4.0/

\begin{abstract}
Using model like hot air bloom with zero-weighted membrane wrapped hot air, surrounded by cold air, this paper sets up a partial differential equation (PDE) of motion of mushroom cloud by modifying Navier-Stokes (N-S) equations. The obtained equation is a vector PDE. It states that the derivative of velocity is with respect to time proportions to the gradient of temperature with respect to trace. Its solution is obtained by the method of separating variables for scalar function. These results have been compared with well agreement with literatures. Highlight: The Principle of Minimum Energy Release (PMER) is used to prove the pulse-mode of explosion of nuclear weapon, as great Earthquake, and optimum path problems.
\end{abstract}

\section{Keywords}

A-Bomb, H-Bomb, Mushroom Cloud, Boyles Law, Chares Law, Mass-Energy Equation, Gravity-Buoyancy Field, Principle of Minimum Energy Release, Principle of Reciprocal Displacement, N-S Equations

\section{Introduction}

Mushroom cloud is a special cloud, created by atomic bomb or nuclear bomb. The Tsar Bomb is the most powerful weapon constructed. The details are described in [1]. There are many studies on A-bomb and H-bomb. However, no literatures on mushroom cloud description by analytic equation can be found. This paper aims to set up an equation on motion of mushroom cloud using model as [2], scientific laws, and modifying Navier-Stokes equations (N-S equations). The obtained equation is a vector partial deferential equation (PDE). It states that the derivative of velocity is with respect to time proportions to the gradient of temperature with respect to trace. The obtained solution is solved by the method of separating variables. This obtained equation and its solution have 
been compared with wind speed equation of a point (mass) in air [3] and its solution [4].

In Section $\$ 2$, the methods of analysis include $\$ 2.1$ Coordinates and basic hypotheses; $\$ 2.2$ Model of mushroom cloud; $\$ 2.3$ Scientific laws; $\$ 2.4$ The gravity-buoyancy field; $\$ 2.5$ The Principle of Minimum Energy Release (PMER); $\$ 2.6$ The Principle of Reciprocal Displacement (PRD). The purpose of this paper is to set up an equation of motion of mushroom cloud, to find the solution, and to use applications of PMER, etc.

In Section $₫ 3$, Set up PDE of mushroom cloud by modifying N-S equations.

Section $\$ 4$, is Solution of the PDE of mushroom cloud (3-12) by method of separating variables.

Section $₫ 5$, is Checking the obtained PDE and its solution.

Section $\$ 6$, is Discussion.

Finally, a conclusion is made.

\section{Method of Analysis}

\subsection{Coordinates and Basic Hypotheses}

\section{Coordinates:}

Cylindrical coordinates: Let $(r, \vartheta, z)$ be the cylindrical coordinates with $O(0,0,0)$ at the explosion center. The relation between $(x, y)$ and $(r, \vartheta)$ is:

$$
\left\{\begin{array}{l}
x=r \cos \vartheta, \\
y=r \sin \vartheta,
\end{array} \quad(0 \leq \vartheta \leq 2 \pi, 0 \leq r<\infty,-\infty<z<\infty)\right.
$$

$(\boldsymbol{i}, \boldsymbol{j}, \boldsymbol{k})$ and $\left(\boldsymbol{e}_{r}, \boldsymbol{e}_{\theta}, \boldsymbol{k}\right)$ denote the unit vectors of Cartesian and cylindrical coordinates respectively. In the following, Bold face denotes vector, matrix, tensor; Non-bold face represents scalars. For z-axis-symmetry, a point $f(r, \vartheta, z)$ independents to $\vartheta$ and can be simplified by $f(r, z)$.

\section{Basic hypotheses:}

1) The motion of mushroom cloud is z-axis-symmetric.

2) The air is continuous, isotropic and incompressible. No overlap or vacuum exists.

3) The gravity-buoyancy field holds in the whole atmosphere, where the forces anti-gravity or the order of equilibrium state are called "buoyancy". The mass of buoyancy (e.g., mass of hot air, mass of medium) is considered as negative mass, with a minus sign. The remainder is considered as positive mass. The positive mass attracts each other; The negative mass attracts each other. Different signed masses are rejected each other.

\subsection{Model of Mushroom Cloud}

Like case-in-case in detective story, model-in-model had been used in author's paper [2], where a bigger model involves smaller model. The bigger model likes a hot air bloom, (in the following it is called "bloom") surrounding by cold air. The smaller model (hot air) moves up-side-down cyclically in the bloom axis-symmetrically. In this paper, only the motion of the bloom is studied. 
The reason for why the weight of membrane is designed to be approached to zero, as stated by the author in [2] [3] that in fact the air is not wrapped by anything. The design of air wrapped by a zero-weighted membrane is to give the air with shape and volume so that the scientific laws, e.g., Boyle's law and Chares law etc. can be used to form an equation of air motion.

The membrane can be viewed as the boundary between the hot air and cold air.

\subsection{Scientific Laws}

The scientific laws related to substances involving in mushroom cloud. Different substance has different properties relating to different scientific laws. For example, for cloud droplet, for PM 2.5, etc. have scientific laws related to their properties [2]. In the following, only major scientific laws are mentioned and they can be used in combination.

1) The combination of Boyles law and Chares law (2.3-1), which has advantage over the ideal gas law (2.3-2).

$$
p V=R T \text {, }
$$

where $p$ is the pressure, acting normal to the boundary of "container" ("bloom"), inside the boundary; $V$ is the volume of the container, inside the boundary.

The dimensions on both sides of (2.3-1) must be the same, then, the dimension of constant $R$ is $\left(\mathrm{J} /{ }^{\circ} \mathrm{C}\right)$, where $\mathrm{J}$ is Joule.

The ideal gas law was first stated by Clapeyrong, $\mathrm{E}$ in 1834, as a combination of Bole's law, Chares/ law, and Avogadro's law [5].

$$
p V=n R_{g} T \text {, }
$$

$R_{g}$ is gas constant, equal to the product of Bolzmann constant and Avogadro constant. Equation (2.3-2) can be derived empirically or by kinetic theory under some assumptions including moles random motion, etc. The element of the ideal gas law is moles or atom, its size belongs to micro-level. If one tries to use a micro-level law with random motion assumption to a problem of macro-level, then, even if one got a result, the result must belong to uncertain type. Therefore, we do not use the ideal gas law while prefer to use the combination of Boyle's la and Chares' law.

\section{2) The mass-energy equivalence [6]}

The mass-energy equivalence, proposed by Albert Einstein in 1905 and has been studied widely and deeply in the world [6]. For keeping two terms of Taylor expansion of object in low speed motion, we have [6]:

$$
E=m_{0} c^{2}+\frac{1}{2} m_{0} u^{2},
$$

where $m_{0}$ represents rest mass, $c$ is the velocity of light $(c=299,792,458 \mathrm{~m} / \mathrm{s}), u$ is the velocity of the object. The term $m_{0} c^{2}$ is a constant, it does not affect the behavior of the object, therefore we can move it to the left hand side, and re-write as: 


$$
E_{0}=m_{0} u^{2}
$$

where $E_{0}$ represents the total energy (including potential energy, kinetic energy, electromagnetic energy, light radiation, heat energy, etc. i.e.,

$$
E_{0}=E_{p}+E_{k}+E_{T}=2 E_{k},
$$

where $E_{p}$ is the potential energy in gravity-buoyancy field; $E_{k}=(1 / 2) m_{0} u^{2}$ is the kinetic energy including impact wave's energy; $E_{T}$ is the remained energy including heat, electromagnetic, light radial emission energy. From (2.3-4), we have:

$$
E_{k}=E_{p}+E_{T}
$$

Since the calculation of $E_{T}$ is difficult, (2.3-5) gives an easier way for $E_{T}$ calculation.

3) Combination of Boyle's law, Chares' law and Einstein's mass-energy equation.

Since both dimensions of combination of Boyle's law and Chares' law and Einstein mass-energy equation are the same, (i.e., N.m or J), therefore, we can combine these laws into one equation, i.e., (2.3-1) equals (2.3-3), then, we have:

$$
p V=R T=E=\lim _{u \rightarrow c} m u^{2},
$$

Once the released energy $E$ of the nuclear bomb is given, the temperature $T$ and $p V$ can be calculated by (2.3-6). In order to obtain an equation linking $p$ and $V$, we use the Green formula [7].

Green formula:

$$
\iint_{s} \varphi \nabla \psi \cdot \mathrm{d} s=\iiint_{V}(\varphi \cdot \psi+\nabla \varphi \cdot \nabla \psi) \mathrm{d} V,
$$

where $S$ is the curve surface of $\delta \aleph ; V$ is the volume of $\aleph ; \varphi$ and $\psi$ are two scalar functions, they have continuous partial derivative in $s$ and two order continuous partial derivatives in $V ; \nabla=\boldsymbol{i} \frac{\partial}{\partial x}+\boldsymbol{j} \frac{\partial}{\partial y}+\boldsymbol{k} \frac{\partial}{\partial z}$ is the Hamilton operator (or del operator); $\boldsymbol{i}, \boldsymbol{j}, \boldsymbol{k}$ are unit vectors of Cartesian coordinates; $\Delta=\nabla \cdot \nabla=\nabla^{2}$ is the Laplace operator.

Let $\varphi=p$ and $\nabla \psi=V$, then (2.3-7) becomes:

$$
\begin{aligned}
\iint_{S} E \mathrm{~d} s & =\iint_{S} p V \mathrm{~d} s=\iiint_{V}(p \nabla V+V \nabla p) \mathrm{d} V=\iiint_{V} \nabla(p V) \mathrm{d} V \\
& =R \iiint_{V} \nabla T \cdot \mathrm{d} V=\iiint_{V} \nabla E \cdot \mathrm{d} V=R \iint_{S} T \cdot \mathrm{d} s
\end{aligned}
$$

\section{4) Newton's second law}

$$
\boldsymbol{F}=\boldsymbol{m} \cdot \boldsymbol{a}=\boldsymbol{m} \cdot(\mathrm{d} \boldsymbol{u} / \mathrm{d} t),
$$

The Newton's second law describes motion of point mass or solid mass, where mass $\mathrm{m}$ is considered as a constant. However, for fluid substance, since the shape and volume can changeably and the mass can also change due to growth, removal, coagulation of substance. Therefore, the fluid mass is considered as a variable vector as shown in [2]. 


\subsection{Gravity-Buoyancy Field}

In gravity-buoyancy field, gravity acts vertically on cold air; while buoyancy acts vertically and horizontally on hot air.

\section{1) Potential energy $E_{p}$}

The capability of work done by position changing of object is called the potential energy

$$
E_{p}=\iiint_{V}\left[-\rho_{c} g z+\rho_{h}\left(\mathrm{~d} u_{z} / \mathrm{d} t\right) z+0-\rho_{h}\left(\mathrm{~d} u_{r} / \mathrm{d} t\right) r\right] \mathrm{d} v,
$$

where $\mathrm{d} m_{c}=\rho_{c} \mathrm{~d} v, \quad \mathrm{~d} m_{h}=\rho_{h} \mathrm{~d} v$, and $\rho_{h}, \rho_{c}$ are the masses, and density of hot air and cold air of $\mathrm{d} v$, respectively; $g$ is the gravity acceleration (opposite to the displacement $z$ ); $V$ is the volume of bloom; $u_{z}$ and $u_{r}$ are the components of the velocity $u$ in $z$-direction and $r$-direction of the bloom, respectively; $\boldsymbol{u}=u_{r} \boldsymbol{e}_{r}+u_{z} \boldsymbol{K}$, or $u^{2}=u_{r}^{2}+u_{z}^{2} . E_{p}$ is the potential energy of the bloom. Where the first term shows the work done by force on opposite direction of displacement and is defined as negative work. The second term shows the work done by force on same direction of displacement $z$. The third term 0 shows that gravity does not act on the horizontal level of hot air. The fourth term shows the work done by horizontal buoyancy, which against the direction of horizontal displacement due to denotation.

\section{2) The kinetic energy $E_{k}$}

$$
E_{k}=(1 / 2)\left(m_{c}+m_{h}\right) u^{2},
$$

3) The heat and other energies $E_{T}$

$$
E_{T}=E-E_{p}-E_{k}=E_{k}-E_{p},
$$

\subsection{Principle of Minimum Energy Release (PMER) [8]}

The Principle of minimum energy release (PMER) had been stated and used in [8] simply. Here, we re-stare it more clearly.

The Principle of energy release (PMER) states that a path connected points A and $B$, and an object goes from $A$ to $B$ with releasing energy proportioning to the square of velocity on the path; there are many possible paths to reach B from A, the actual carried out (or the best) path is that one which releases minimum total energy.

1) Now we use PMER to prove the mode of energy release by nuclear fusion is a pulse-mode

Rewrite (2.3-6) as

$$
Y=\Delta E=m\left(u^{2}\left(t_{1}\right)-u^{2}\left(t_{0}\right)\right),
$$

where $Y$ represents energy release, $u \rightarrow c$ is the velocity, $t_{1}$ and $t_{0}$ are the time of end and beginning of the explosion respectively.

Construction a paths function $p(t)=m u^{2}(t)$, such that

$$
Y=\int_{t_{0}}^{t_{1}} p(t) \mathrm{d} t=\int_{t_{0}}^{t_{1}} m u^{2}(t) \mathrm{d} t,
$$

There are many paths $p(t)$ satisfying (2.5-1). For example, a straight line or 
a curve line from $t_{0}$ to $t_{1}$. The actual one must be that with minimum energy release.

\section{Proof:}

This is an optimization problem, i.e.,

$$
\min _{p} Y=\min _{u} Y=\min _{u} \int_{t_{0}}^{t_{1}} m u^{2}(t) \mathrm{d} t,
$$

The necessary condition for the problem to be minimum is:

$$
\mathrm{d} Y / \mathrm{d} u=0,
$$

Then, we have

$$
2 m \int_{t_{0}}^{t_{1}} u(t) \mathrm{d} t=0
$$

Since $t_{0} \neq t_{1}$, and $t_{1}$ can be arbitrarily chosen, by Newton-Leibniz formula, the integrand must be zero, then, we have:

$$
u(t)=0,\left(t_{0} \leq t<t_{1}\right) \text { and } u\left(t_{1}\right)=c,
$$

Equation (2.5-6) shows that the path is a broken line, $u(t)=0,\left(t_{0} \leq t<t_{1}\right)$ and $u\left(t_{1}\right)=c$. This is a pulse-mode, the velocity suddenly increases to infinity at $t=t_{1}$. Like a great earthquakes releasing energy in a very short time [8], the detonation releasing energy is also in a pulse-mode.

According to the data of the time interval of denotation releasing more than $2.1 \times 10^{5} \mathrm{TJ}$ within $39(\mathrm{~ns})\left(=3.9 \times 10^{-10} \mathrm{~s}\right)$ [9], which confirms that the denotation is in a pulse-mode.

The PNER is similar to the Principle of virtual displacement (or the Principle of possible displacement) in mechanics, which states that in all possible displacements which satisfies equilibrium equation and boundary conditions, the actual one of displacements that happened is that which releases minimum energy.

The PNER could be used to strategy selection of sport games on path with energy releasing proportioning to the square of velocity. People often see that in a long running or swimming, sports usually release minimum energy in early stage but pulse release the total energy at end stage.

\section{2) Principle of reciprocal displacements (PRD)}

When a group of masses $m_{h}$ are moving from location A to location B, meanwhile the masses $m_{c}$ in B will removal and coagulation to the "vacuum" of A, i.e., the vacuum must be filled by nearby masses $m_{c}$, due to continuous assumption.

The PRD states that in gravity-buoyancy field, $\aleph_{h}(\mathrm{~A})$ moves to $\aleph_{c}(\mathrm{~B})$, meanwhile, $\aleph_{c}(\mathrm{~B})$ moves to $\aleph_{h}(\mathrm{~A})$.

\section{Proof:}

Since masses group $\aleph_{h}$ occupied B, the masses group $\aleph_{c}$ must be driven out from B, meanwhile the "vacuum" of A must be filled by nearby masses. There are many paths of masses $\aleph_{c}$ in everywhere to fill the "vacuum" of A, by the Principle of Virtual Displacement (PVD), the actual one path is that which 
releases minimum energy, that is: $\aleph_{c}(\mathrm{~B})$ moving to $\aleph_{h}(\mathrm{~A})$ directly. Because the straight line is shortest and masses $\aleph_{c}$ moving from $B$ to A leases minimum energy.

The PRD can be viewed as PMER used for path of re-filled of vacuum rooms.

\section{Set Up PDE of Mushroom Cloud by Modifying N-S Equations}

The N-S equations are a set of PDE that describe incompressible fluids and have widely used in weather, ocean currents, water flow in a pipe, air flow around a wing, etc. [10]. They are also of great interest to pure mathematics. The Clay Mathematics Institute has offered "Millennium Prize Problem" [11] to the existence and smoothness of the solution of N-S equations. However, non-existence of solution for general N-S equations has been proved [12]. Nevertheless, modifying the N-S equations may be used to describe the motion of mushroom cloud. There are five reasons to modify the $\mathrm{N}-\mathrm{S}$ equations:

1) The N-S equations do not consider the affection of buoyancy. For many cases of practical application (e.g., rocket, aircraft, etc.), the affection of buoyancy can be neglected. However, for atmosphere the buoyancy has the same importance as gravity.

2) The gravity field needs to change to the gravity-buoyancy field.

3) For high speed motion as denotation, the drag force needs to modify.

4) For atmosphere, the temperature must be taken into account.

5) The N-S equations dealing with a point mass in $3 \mathrm{D}$ space needs to include a group of points masses.

The general form of $\mathrm{N}-\mathrm{S}$ equations is [10]:

$$
\rho\left(\frac{\partial \boldsymbol{u}}{\partial t}+\boldsymbol{u} \cdot \nabla \boldsymbol{u}\right)=-\nabla p+\nabla \cdot T_{s}+\boldsymbol{f},
$$

where $\boldsymbol{u}$ is the flow velocity; $\rho$ is the fluid density; $p$ is the pressure; $\boldsymbol{T}_{s}$ is the (deviatoric) stress tensor; $\boldsymbol{f}=\rho \boldsymbol{g}$ represents the body force per unit volume acting on the fluid; $\nabla$ is the del (or Hamilton) operator. For Cartesian coordinates,

$$
\boldsymbol{\nabla}=\boldsymbol{i} \frac{\partial}{\partial x}+\boldsymbol{j} \frac{\partial}{\partial y}+\boldsymbol{k} \frac{\partial}{\partial z}
$$

For z-axis-symmetry, the cylindrical coordinates' del operator $\nabla_{c}$ is [7]:

$$
\begin{gathered}
\nabla_{c}=\boldsymbol{e}_{r} \frac{\partial}{\partial r}+\boldsymbol{e}_{\theta} \frac{1}{r} \frac{\partial}{\partial \theta}+\boldsymbol{k} \frac{\partial}{\partial z}=\boldsymbol{e}_{r} \frac{\partial}{\partial r}+\mathbf{0}+\boldsymbol{k} \frac{\partial}{\partial z}, \\
\boldsymbol{u}=u_{r} \boldsymbol{e}_{r}+u_{z} \boldsymbol{k},
\end{gathered}
$$

The modified N-S equations for mushroom cloud:

At first, we simplify (3-1):

$$
\rho\left(\frac{\partial \boldsymbol{u}}{\partial t}+\boldsymbol{u} \cdot \nabla_{h} \cdot \boldsymbol{u}\right)=-\nabla p+0+\rho \boldsymbol{g},
$$

where the stress tensor $\boldsymbol{T}_{s}$ is zero, since the viscosity of air can be neglected; 
and the

$$
\boldsymbol{u} \cdot \nabla_{h} \cdot \boldsymbol{u}=\left|\begin{array}{ccc}
u_{r} & 0 & u_{z} \\
\frac{\partial}{\partial r} & 0 & \frac{\partial}{\partial z} \\
u_{r} & 0 & u_{z}
\end{array}\right|=0
$$

where the inner product of three vectors $(\boldsymbol{a} \cdot \boldsymbol{b} \cdot \boldsymbol{c})$ is defined [7]:

$$
\begin{gathered}
(\boldsymbol{a} \cdot \boldsymbol{b} \cdot \boldsymbol{c}) \equiv\left|\begin{array}{lll}
a_{x} & a_{y} & a_{z} \\
b_{x} & b_{y} & b_{z} \\
c_{x} & c_{y} & c_{z}
\end{array}\right|, \\
\boldsymbol{a}=a_{x} \boldsymbol{i}+a_{y} \boldsymbol{j}+a_{k} \boldsymbol{k}, \quad \boldsymbol{b}=b_{x} \boldsymbol{i}+b_{y} \boldsymbol{j}+b_{z} \boldsymbol{k}, \boldsymbol{c}=c_{x} \boldsymbol{i}+c_{y} \boldsymbol{j}+c_{z} \boldsymbol{k} .
\end{gathered}
$$

And $\left(\boldsymbol{u} \cdot \nabla_{c} \cdot \boldsymbol{u}\right)=0$, since two rows in the determinant are the same.

Now, (3-5) becomes:

$$
\rho \frac{\partial \boldsymbol{u}}{\partial t}=\nabla_{c} p+\boldsymbol{g} \rho,
$$

Equation (3-8) holds for a point (mass) in air, which states that the derivative of velocity respect to time proportions to the gradient of pressure respect to trace.

In order to change the relationship between $u$ and $p$ to the relationship between $u$ and $T$, and suits for the case of volume $V=V(T, t) \neq 0 \quad$ (not the case of a point, and $V$ is the function of temperature and time, but independent of space $r$, and $z$ ), integrals both side of (3-8), we have:

$$
\iiint_{V} \rho\left[\frac{\partial \boldsymbol{u}}{\partial t}\right] \mathrm{d} v=\iiint_{V}\left[\nabla_{c} p+\boldsymbol{g} \rho\right] \mathrm{d} v,
$$

where $V$ is the volume of the container (bloom). By theorem of mean value of integration, we have:

$$
\frac{\partial \boldsymbol{u}}{\partial t} \rho V=\nabla_{c} p V+\boldsymbol{g} \rho V,
$$

Notes that $V \nabla_{c} p=\nabla_{c}(p V)=R \nabla_{c} T$, and $m=\rho V$, then, (3-10) becomes:

$$
\frac{\partial \boldsymbol{u}}{\partial t} m=R \nabla_{c} T+m \boldsymbol{g},
$$

Second, instead of $m$ by $m_{h / c}=m_{h} / m_{c}$, then, (3-11) becomes:

$$
\frac{\partial \boldsymbol{u}}{\partial t}=k \nabla_{c} T_{h / c}+\boldsymbol{g}, \quad\left(k=R m_{c} / m_{h}\right) \quad(3-12)
$$

Similarly, (3-8) becomes:

$$
\frac{\partial \boldsymbol{u}}{\partial t}=\frac{m_{c}}{m_{h}} \nabla_{c} p_{h / c}+\boldsymbol{g}
$$

where $p_{h / c}=p_{h} / p_{c}, T_{h / c}=T_{h} / T_{c}$. The reason for why we do this instead is because of the gravity-buoyancy (hot air mass/cold air mass) co-exists

Equation (3-12) is the PDE for the total mass in bloom. It shows that the derivative of mean value of velocity vector respect to time proportions to the gra- 
dient of temperature $T_{h / c}$ respect to trace of motion.

Equation (3-13) is the PDE for the total mass in bloom. It shows that the derivative of the mean value of velocity vector respect to time, proportions to the gradient of pressure $p_{h / c}$ respect to trace of motion.

\section{Solution of the PDE of Mushroom Cloud (3-12) by Method of Separating of Variables}

The method of separating variables is one of methods for solving PDE of scalar function. If for PDE of vector function, it is more complicated. However, for the motion of mushroom cloud due to denotation, the moving path is a straight line (the $\mathrm{z}$-axis), not a curve. Therefore the vector function can be simplified to scalar function.

Rewrite (3-12), the vector term is changed to scalar term, we have

$$
\frac{\partial \boldsymbol{u}}{\partial t}=k \nabla_{c} T+g,
$$

where $T_{h / c}$ is simplified by $T$. Let

$$
u_{1}(s, t)=u(s, t)+g t,
$$

Suppose that the variables $s=s(r, z)$ and $t$ of $\boldsymbol{u}_{1}(s, t)$ and $T=T(s, t)$ can be separated. Let

$$
\begin{aligned}
& u_{1}(s, t)=a(s) b(t), \\
& T(s, t)=c(s) f(t),
\end{aligned}
$$

Substituting (4-2)-(4-4) into (4-1), we have:

$$
\frac{\mathrm{d} \boldsymbol{b}(t)}{\mathrm{d} t} a(s)=k\left[\frac{\partial c(s)}{\partial r}+\frac{\partial c(s)}{\partial z}\right] f(t),
$$

Separating variables, we have

$$
\frac{\mathrm{d} \boldsymbol{b}(t)}{f(t) \mathrm{d} t}=k \frac{\left[\frac{\partial c(s)}{\partial r}+\frac{\partial c(s)}{\partial z}\right]}{a(s)},
$$

In order to make the solution simple, let $b(t)=f(t), a(s)=k c(s)$, then, (4-6) becomes:

$$
\frac{\mathrm{d} \boldsymbol{b}(t)}{\boldsymbol{b}(t) \mathrm{d} t}=k \frac{\nabla_{c} a(s)}{a(s)}=\mathrm{const},
$$

If (4-7) holds, then, each side must be a constant, i.e.,

$$
\begin{gathered}
\frac{\mathrm{d} \boldsymbol{b}(t)}{\boldsymbol{b}(t)}=A_{1} \mathrm{~d} t, \quad\left(A_{1}=\text { const }\right) \\
k \nabla_{c} a(s) / a(s)=B, \quad(B=\text { const })
\end{gathered}
$$

Or

$$
\frac{\partial a(s)}{a(s) \partial r}+\frac{\partial a(s)}{a(s) \partial z}=B / k,
$$


Separating two terms on the left hand side of (4-10), we have:

$$
\begin{aligned}
& \frac{\partial a(s)}{a(s)}=B_{1} \partial r, \\
& \frac{\partial a(s)}{a(s)}=B_{2} \partial z,
\end{aligned}
$$

Integrating both sides of (4-8), (4-11), (4-12), we have

$$
\begin{gathered}
b(t)=A \exp \left[A_{1} t\right], \\
a(s)=A_{2} \exp \left[B_{1} r+B_{2} z\right],
\end{gathered}
$$

Substituting (4-13), (4-14) and (4-3) into (4-2), we have

$$
u(s, t)=A A_{2} \exp \left[B_{1} r+B_{2} z+A_{1} t\right]-g t,
$$

Substituting $b(t)=f(t), a(s)=k c(s)$, into (4-4), we have

$$
T(s, t)=A A_{2} k \exp \left[B_{1} r+B_{2} z+A_{1} t\right],
$$

Equations (4-15) and (4-16) are the solutions of PDE (3-12), where integral constants are determined by initial and boundary conditions.

The solution of PDE (3-13) is similar.

\section{Checking the Obtained PDE and Its Solution}

The obtained equations (3-12), (3-13) are vector PDE. These PDE are similar to the PDE of a point mass in air obtained in [3] by the author, which revealed that the derivative of wind speed respect to time plus a constant is proportional to the derivative of temperature respect to the trace.

Equation (3-12) and (3-13) are derived based on modified N-S equation, scientific laws and gravity-buoyancy field. The equation in [3] is derived for a point mass in air without considering buoyancy in air, based on equilibrium equation, Newton's second law, Boyle's law and Chares' law.

The solution of vector PDE (3-12) and (3-13) is solved by method of separating of variables for scalar function, since the trace is a straight line (along the z-axis). Similarly, the solution of PDE in [3] is also solved by method of separating of variables in [4]. Since the equation is a vector PDE, it cannot use the method of separating variables for scalar function, unless the trace is a straight line. If the trace is a curve, it can be treated as segmental straight lines.

The equations (3-12) and (3-13) and their solutions can be compared with each other in [3] and [4]. The mushroom cloud is visible, while the air substance motion in air is un-visible. Therefore, the equations (3-12) and (3-13) and their solutions help the equation in [3] and solutions in [4] to be more believable.

\section{Discussion}

$\mathrm{Q}=$ question, $\mathrm{A}=$ answer.

Q1: Why study the motion of mushroom cloud?

A: Because the video of motion of mushroom cloud is visible, while the mo- 
tion of air substance in air is often difficult to visible. We can check the accuracy of calculating results of mushroom cloud by comparing with others.

\section{Q2: How to study the motion of mushroom cloud?}

A: 1) using a model of zero-weighted "bloom" wrapped hot air, so as to give the "bloom" has shape and volume, such that scientific laws can be used.

2) The "bloom" is located in gravity-buoyancy field surrounded by cold air.

3) Using scientific laws to find solution of problems.

\section{Q3: Is there any problem related to motion of mushroom cloud?}

A: The problem of "hot air bloom", weather detecting bloom, bubble in water, etc. are related problems.

\section{Conclusions}

Using the model of zero-weighted membrane to wrapped hot air, together with scientific laws, gravity-buoyancy field, and modifying N-S equations, this paper derives a vector PDE for motion of mushroom cloud. Its solution is obtained by the method of separating variables. Both PDE and its solution are compared with well agreement with literatures.

Highlight: the pulse-mode of denotation of nuclear weapon is proved by Principle of Minimum Energy Release (PMER), as great Earthquake.

\section{Conflicts of Interest}

The author declares no conflicts of interest regarding the publication of this paper.

\section{References}

[1] http://www.tsarbomba.org/

[2] Yun, T.-Q. (2017) New Dynamic Equations of Aerosol in Air of Certain Type. Atmospheric and Climate Science, 7, 551-524. https://doi.org/10.4236/acs.2017.74037

[3] Yun, T.-Q. (2016) Wind Speed Equation of a Point in Air. Fundamental Journal of Modern Physics, 9, 57-64.

[4] Yun, T.-Q. (2016) Existence and Solution of Wind Speed Equation of a Point in Air, British Journal of Mathematics and Computer Science, 17, Article No. 27054, 1-5. https://doi.org/10.9734/BJMCS/2016/27054

[5] Clapeyrong, E. (1834) Memcire sur la puissance motrice de la chuleur. Journal de I'Ecole Polytechnique, XIV, 153-190. (In French)

[6] https://encyclopedia.thefreedictionary.com/mass-energy+equation

[7] Editors of <Mathematical Hand Book> (1979) Mathematical Hand Book. High Education Publishers, Beijing, 446. (In Chinese)

[8] Yun, T.-Q. (2019) Earthquake Fastens Earth Rotation. Asian Journal of Geological Research, 2, Article No. AJOGER.494571-9.

[9] https://沙皇炸弹-360 百科 (In Chinese).

[10] https://doc.wendoc.com/b728ab7ee66381833293fa851.html

[11] https://www.claymath.org/millennium-problem/Navier-Stockes-equation

[12] Yun, T.-Q. (2020) Non-Existence of Solution of Rotation Flow in N-S Equations. 
Global Journal of Pure and Applied Mathematics, 16, 651-655.

http://www.ripublication.com/gjpam.htm

https://doi.org/10.37622/GJPAM/16.5.2020.651-655 